\title{
Predictive Analysis for Radiated Electromagnetic Disturbance in MMC-HVDC Valve Hall
}

\author{
Guishu Liang and Ruimin Zhu
}

\begin{abstract}
The radiated electromagnetic interference (EMI) in the valve hall of the modular multilevel converter (MMC) station must be limited below a certain value to ensure the normal operation of the equipment and the safety of the engineers. In this paper, a method for predicting the radiated EMI is proposed, which takes all the necessary factors, such as the physics-based characteristics for IGBT module, sub-module topology, and converter space structure into consideration. This method involves the improved physical model of semiconductor devices, which discards the non-physical feedback parameter introduced by some previous research. At the same time, through the decoupling of the submodule and the bridge arm circuit, the order of the large-scale system is reduced so that the sub-modules can be calculated in parallel, greatly reducing the overall calculation burden of the model and accelerating the nonlinear small time-step simulation. The wideband characteristics of the converter valve tower are fully considered, with each of the bridge arms regarded as a two-port network with independent sources. The parameter integration and distribution process can also achieve complete parallel calculation. The converter valve tower is modeled as a complex antenna structure with the output voltage of each sub-module as the excitation, the calculation of near-field radiation intensity for the converter valve tower is performed in Alteir ${ }^{\circledR}$ FEKO. The measurement conducted inside an actual 49-level converter station verifies the accuracy of the model.
\end{abstract}

Index Terms-High-voltage direct-current (HVDC), insulated gate bipolar transistor (IGBT), modular multilevel converter (MMC), radiated electromagnetic interference (EMI), wide-band modeling.

\section{INTRODUCTION}

$\mathrm{M}$ ODULAR multilevel converters (MMC) are presently the high-priority option of converter topology for voltage source converter high voltage direct current (VSCHVDC) transmission project due to their high efficiency and flexible operation mode [1]-[4]. Concomitantly, the relevant electromagnetic interference (EMI) characteristics, especially

Manuscript received Janurary 15, 2020; revised March 8, 2020; accepted May 27, 2020. Date of publication June 30, 2020; date of current version June 16, 2020. This work was supported by the National Natural Science Foundation of China under Grant 51207054.

G. Liang is with the School of Electrical and Electronic Engineering, North China Electric Power University, Baoding 071003, China (e-mail: gshliang@263. net).

R. Zhu is with the School of Electrical and Electronic Engineering, North China Electric Power University, Beijing 102206, China, also with the Department of Electrical and Computer Engineering, University of Alberta, Edmonton, Alberta T6G 2V4, Canada (e-mail: zruimin@ualberta.ca).

Digital Object Identifier 10.24295/CPSSTPEA.2020.00011 the pre-cautionary EMC validation during the planning stage, have become a burning issue for the converter design and project construction. The semiconductor devices are the major interference sources due to the high-frequency component in the voltage and current generated during their commutation [5], [6], propagating through the parasitic paths, and then emitting high-frequency electromagnetic wave into the surrounding space. The suppression of radiated EMI is essential and should be addressed as early as possible in the design stage.

Radiated EMI from a power electronic converter is a comprehensive and challenging issue that relates to parasitic effects, circuit topology, and component layout within the device itself, in conjunction with the connected system in which it operates. From the perspective of electromagnetic radiation, the MMC valve tower is essentially an antenna with complex space topology. The measurement of the electro-magnetic environment was conducted in some actual MMC-HVDC converter stations [7], [8]. However, the method has the inherent deficiency for its dependency on measurement, which implies that it won't be applicable until the device under test is manufactured. Thus, the measurement-based approach should only be regarded as the last validation stage or a part of the painstaking trial and error process in which the experience of the designer is essential [9] since it fails to provide any reliable information on what changes should be made if the noise level does not meet the standard requirements. Thus, a veritable predictive method that is competent to guide in the design stage of the project is urgently needed.

The key to a successful prediction for the radiated EMI lies in the excitation extraction and the modeling of the equivalent antenna structure, which involves a thorough insight into the converter topology, operation principles, and parasitic parameters. Actually, it can be concluded that radiated EMI characterization is inseparable from conducted EMI prediction [10], [11]. The finite-element analysis (FEA) [12] or partial element equivalent circuit (PEEC) [13] method is usually selected to establish the wideband circuit model and carry out the time-domain simulation, in which the nonlinear behavior or physics-based model of semiconductor devices are needed. These approaches are common in the analysis for $2-\mathrm{L}$ or $3-\mathrm{L}$ PWM converters with only a few IGBT modules. However, for the converter topology with numerous switch units like $\mathrm{MMC}$, the computation burden will be extremely large, which will make it impractical.

In this paper, an improved physics-based model of IGBT and diode is proposed, which can be discretized for time- 
domain simulation. Circuit partition method for the MMC valve is applied to split each sub-module (SM) from the bridge arm, thus the complex model of the IGBTs and diodes can be solved within each SM circuits and in a parallel manner. This method of segmentation divides the whole system into small-size sub-circuits, which helps to improve the numerical stability and speed up the convergence process [15]. GPU's massive parallelism is utilized to expedite the simulation of MMC-HVDC system EMI characteristics [16]. The stray capacitance inside the converter tower can be merged with the SM output voltage so that the bridge arm is actually a two-port network with independent sources inside. The similarity of the fine-grained network enables programming the algorithm in the single instruction multiple data (SIMD) format by the CUDA C language and parallel execution by corresponding kernels with numerous threads. The antenna model of a converter valve tower from an offshore wind farm HVDC link project is established in Alteir ${ }^{\circledR}$ FEKO according to the actual structure and size. The voltage of the SMs from the timedomain simulation is used as the excitation of the 3D antenna. The radiated EMI is measured in the valve hall and the comparative analysis was performed to evaluate the accuracy of the proposed method.

The paper is organized as follows: Section II shows the improved IGBT/diode model and the method of the parasitic parameters extraction; Section III presents the detailed partitioning methodologies of the GPU accelerated simulation framework. Section IV shows the antenna model and experimental verification of the method. Finally, Section V gives the conclusion of this paper.

\section{Wide-BAnd Modeling OF VALVE Tower}

\section{A. Improved IGBT and Diode Pair Physics-Based Model}

The switching of IGBT and power diode involves the drift and diffusion of carriers, which is often described by the 1-D ambipolar diffusion equation (ADE). Linear distribution simplification [17] of the carriers can achieve significant facilitation of the solving process and get the circuit model. Some other numerical methods provide the solution of the ADE by the methods of Fourier series decomposition [20], finite element [21], and finite difference method [22], in which a non-physical factor has to be introduced when the large coefficient $K$ is used to model the depletion of the carriers at the junction.

An improved physics-based model for IGBT is proposed based on the finite element method to make the solution fullphysical and more theoretically acceptable. The proposed model takes into account emitter recombination in the highly doped end regions, conductivity modulation in the epilayer, carrier-carrier scattering, and the moving boundaries effect during reverse recovery [22], showing good convergence properties and fast simulation times.

IGBT Model: The IGBT can be deemed as a Darlington combination of MOSFET and BJT as shown in Fig. 1. The gate current of the MOSFET is [17]:

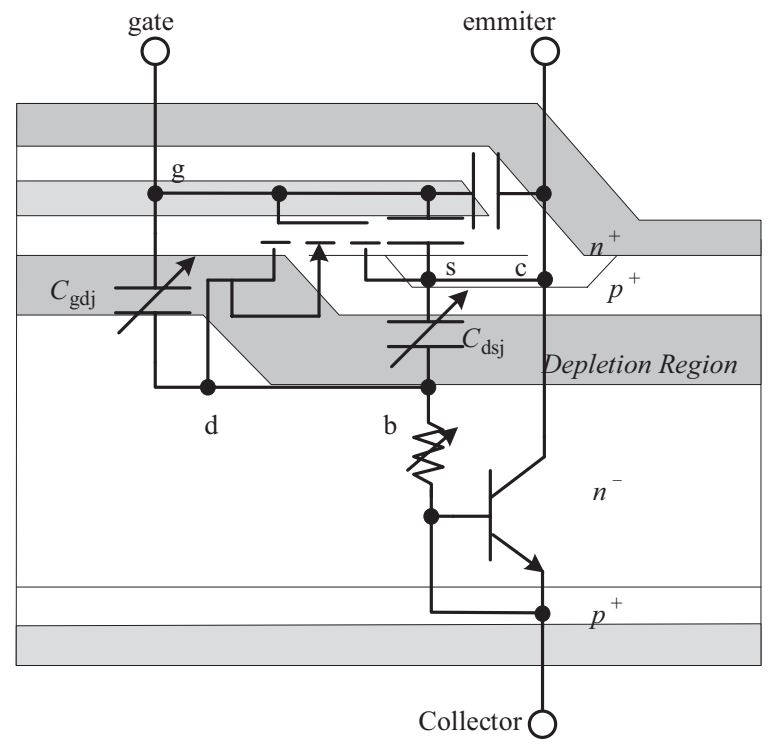

Fig. 1. Physics-based IGBT model.

$$
\frac{\mathrm{d} v_{\mathrm{gs}}}{\mathrm{d} t}=\frac{I_{\mathrm{g}}}{C_{\mathrm{gs}}+C_{\mathrm{gd}}}+\frac{C_{\mathrm{gd}}}{C_{\mathrm{gs}}+C_{\mathrm{gd}}} \frac{\mathrm{d} v_{\mathrm{ds}}}{\mathrm{d} t}
$$

where $C_{\mathrm{gs}}, C_{\mathrm{ds}}$, and $C_{\mathrm{gd}}$ are the gate-source, collector-base, and the gate-drain junction capacitance, which are given as:

$$
\begin{gathered}
C_{\mathrm{bcj}}=\frac{A \epsilon}{\sqrt{\frac{2 \epsilon\left(v_{\mathrm{ds}}+0.6\right)}{1 N_{L}}}} \\
C_{\mathrm{dsj}}=\frac{\left(A-A_{\mathrm{gd}}\right) \epsilon}{\sqrt{\frac{2 \epsilon\left(v_{\mathrm{ds}}+0.6\right)}{1 N_{L}}}} \\
C_{\mathrm{gdj}}=\frac{\left(A_{\mathrm{gd}}\right) \epsilon}{\sqrt{\frac{2 \epsilon\left(v_{\mathrm{ds}}-v_{\mathrm{gs}}+0.6\right)}{1 N_{L}}}}
\end{gathered}
$$

Carrier distribution in N-region under large injection can be obtained by solving the bipolar transport equation [18]:

$$
\begin{aligned}
& I_{\mathrm{n}}=\frac{b}{1+b} I_{\mathrm{T}}+q A D_{\mathrm{a}} \frac{\partial p}{\partial x} \\
& I_{\mathrm{p}}=\frac{b}{1+b} I_{\mathrm{T}}-q A D_{\mathrm{a}} \frac{\partial p}{\partial x}
\end{aligned}
$$

where $D_{\mathrm{a}}=2 D_{\mathrm{n}} D_{\mathrm{p}} /\left(D_{\mathrm{n}}+D_{\mathrm{p}}\right)$ is the ambipolar diffusion coefficient, $b$ is the ambipolar mobility ratio of $\mu_{\mathrm{n}}$ and $\mu_{\mathrm{p}}$, and $\tau_{\mathrm{hl}}$ is the high-level lifetime. The boundary condition for the equation at $x=x_{1}$ and $x=x_{\mathrm{r}}$ can be written as [20]:

$$
\begin{aligned}
& \frac{\partial p}{\partial t}\left(x_{1}, t\right)=\frac{1}{2 q A}\left[\frac{I_{\mathrm{n}}\left(x=x_{1}\right)}{D_{\mathrm{n}}}-\frac{I_{\mathrm{p}}\left(x=x_{1}\right)}{D_{\mathrm{p}}}\right] \\
& \frac{\partial p}{\partial t}\left(x_{\mathrm{r}}, t\right)=\frac{1}{2 q A}\left[\frac{I_{\mathrm{n}}\left(x=x_{\mathrm{r}}\right)}{D_{\mathrm{n}}}-\frac{I_{\mathrm{p}}\left(x=x_{\mathrm{r}}\right)}{D_{\mathrm{p}}}\right]
\end{aligned}
$$


where $A$ is the device area, $q$ is the electron charge, $D_{\mathrm{n}}$ and $D_{\mathrm{p}}$ are the diffusion coefficient for electrons and holes respectively. $I_{\mathrm{n}}$ and $I_{\mathrm{p}}$ are the electron and hole current with their relationship expressed as:

$$
\begin{aligned}
& I_{\mathrm{n}}\left(x=x_{1}\right)=q h_{\mathrm{p}} A p_{\left(x=x_{1}\right)}^{2} \\
& I_{\mathrm{p}}\left(x=x_{\mathrm{r}}\right)=q h_{\mathrm{n}} A p_{\left(x=x_{\mathrm{r}}\right)}^{2}
\end{aligned}
$$

In the current MMC, the IGBTs will never be imposed on a negative voltage, thus the $P^{+}-N^{-}$junction is always in the forward bias state and the $N^{-}-N^{+}$junction is always in reverse bias state. The current continuity condition at the junctions are [19]:

$$
\begin{gathered}
I_{\mathrm{T}}=I_{\mathrm{nl}}+I_{\mathrm{pl}} \\
I_{\mathrm{T}}=I_{\mathrm{mos}}+\left(C_{\mathrm{dsj}}+C_{\mathrm{g}} \mathrm{d}\right) \frac{v_{\mathrm{ds}}}{\mathrm{d} t}-C_{\mathrm{gd}} \frac{\mathrm{d} v_{\mathrm{gs}}}{\mathrm{d} t} I_{\mathrm{pr}}
\end{gathered}
$$

where $I_{\mathrm{T}}$ is the total IGBT current, $I_{\mathrm{mos}}$ is the current of the MOS part and also the electron current at the MOS terminal, which is given as:

$$
I_{\mathrm{mos}}= \begin{cases}0, & V_{\mathrm{ds}}<V_{\mathrm{Th}} \\ K_{\mathrm{p}}\left(v_{\mathrm{gs}}-V_{\mathrm{Th}}\right) v_{\mathrm{ds}}-K_{\mathrm{p}} \frac{v_{\mathrm{ds}}^{2}}{2}, & V_{\mathrm{ds}} \leqslant v_{\mathrm{gs}}-V_{\mathrm{Th}} \\ K_{\mathrm{p}} \frac{\left(v_{\mathrm{gs}}-V_{\mathrm{Th}}\right)^{2}}{2}, & V_{\mathrm{ds}} \geqslant v_{\mathrm{gs}}-V_{\mathrm{Th}}\end{cases}
$$

where $V_{\mathrm{Th}}$ is the MOSFET channel threshold voltage, $v_{\mathrm{b}} c$ is the collector-base voltage, $v_{\mathrm{gs}}$ is the gate-source voltage. an additional current component during reverse recovery, due to the charge variations in the space-charge region (this component charges and discharges the anode-base depletion capacitor), $h_{\mathrm{p}}$ and $h_{\mathrm{n}}$ account for emitter recombination effects, and $x_{1}$ and $x_{\mathrm{r}}$ are the left and right borders of the region flooded with excess carriers.

Finite difference substitutions to the ADE are conducted with respect to space, thus the equation for $i_{\text {th }}$ node is [22]:

$$
\begin{gathered}
\left.\frac{\partial p}{\partial x}\right|_{i}=\frac{p_{i+1}-p_{i-1}}{\mathrm{a} \Delta x} \\
\left.\frac{\partial^{2} p}{\partial x^{2}}\right|_{i}=\frac{p_{i+1}-2 p_{i}+p_{i-1}}{\mathrm{a} \Delta x^{2}}
\end{gathered}
$$

Substituting (14) and (15) into (7) and (8), the expression for the $i_{\text {th }}$ node is given as:

$$
\left.\frac{\partial p}{\partial t}\right|_{i}=D_{\mathrm{a}} \frac{p_{i+1}-2 p_{i}+p_{i-1}}{\mathrm{a} \Delta x^{2}}-\frac{p_{i}}{\tau_{\mathrm{hl}}}
$$

and the equation at $x=x_{1}$ and $x=x_{\mathrm{r}}$ are:

$$
\left.\frac{\partial p}{\partial x}\right|_{i=1}=\frac{1}{2 q A}\left(\frac{q A h_{\mathrm{p}} p_{1}^{2}}{D_{\mathrm{n}}}-\frac{I_{\mathrm{T}}-I_{\text {dep }}-q A h_{\mathrm{p}} p_{1}^{2}}{D_{\mathrm{p}}}\right)
$$

$$
\left.\frac{\partial p}{\partial x}\right|_{i=n+1}=\frac{1}{2 q A}\left(\frac{I_{\mathrm{T}}-I_{\text {dep }}-q A h_{\mathrm{p}} p_{\mathrm{n}+1}^{2}}{D_{\mathrm{n}}}-\frac{q A h_{\mathrm{p}} p_{\mathrm{n}+1}^{2}}{D_{\mathrm{p}}}\right.
$$

Thus the equation for all the difference node is expressed as:

$$
\boldsymbol{M} \frac{\partial \boldsymbol{p}}{\partial x}+\boldsymbol{K} \boldsymbol{p}+\boldsymbol{F}=0
$$

where $(n+1) \times(n+1)$ matrix $\boldsymbol{M}, \boldsymbol{K}$, and $(n+1)$-dimensional vector $\boldsymbol{F}$ are given as:

$$
\begin{aligned}
& \boldsymbol{M}=\left[\begin{array}{ccccc}
0.5 & 0 & \cdots & 0 & 0 \\
0 & 1 & \cdots & 0 & 0 \\
\vdots & \vdots & \ddots & \vdots & \vdots \\
0 & 0 & \cdots & 1 & 0 \\
0 & 0 & \cdots & 0 & 0.5
\end{array}\right] \\
& \boldsymbol{K}=\left[\begin{array}{ccccc}
\frac{D_{\mathrm{a}}}{\Delta x^{2}}+\frac{1}{2 \tau_{\mathrm{hl}}} & -\frac{D_{\mathrm{a}}}{\Delta x^{2}} & \cdots & 0 & 0 \\
\frac{D_{\mathrm{a}}}{\Delta x^{2}} & \frac{2 D_{\mathrm{a}}}{\Delta x^{2}}+\frac{1}{2 \tau_{\mathrm{hl}}} & \cdots & 0 & 0 \\
\vdots & \vdots & \ddots & \vdots & \vdots \\
0 & 0 & \cdots & \frac{2 D_{\mathrm{a}}}{\Delta x^{2}}+\frac{1}{2 \tau_{\mathrm{hl}}} & -\frac{D_{\mathrm{a}}}{\Delta x^{2}} \\
0 & 0 & \cdots & -\frac{D_{\mathrm{a}}}{\Delta x^{2}} & \frac{D_{\mathrm{a}}}{\Delta x^{2}}+\frac{1}{2 \tau_{\mathrm{hl}}}
\end{array}\right] \\
& \boldsymbol{F}=\left[\begin{array}{c}
\frac{D_{\mathrm{a}}}{2 q A}\left(\frac{I_{\mathrm{n} 1}}{\mathrm{D}_{\mathrm{n}}}-\frac{I_{\mathrm{T}}-I_{\mathrm{n} 1}}{D_{\mathrm{p}}}\right) \\
0 \\
\vdots \\
0 \\
-\frac{D_{\mathrm{a}}}{2 q A}\left(\frac{I_{\mathrm{mos}}}{D_{\mathrm{n}}}-\frac{I_{\mathrm{p}(n+1)}}{D_{\mathrm{p}}}\right)
\end{array}\right]
\end{aligned}
$$

In the previous references [20]-[22], the voltage $V_{\mathrm{d}}$ across a depletion layer is derived using feedback from the boundary carrier density $p_{\mathrm{x}}$. This establishes a feedback loop that adjusts the boundary position as a function of the blocking voltage $V_{\mathrm{d}}$. The depletion voltage is equal to 0 when the doping concentration is positive, and $V_{\mathrm{d}}$ can be any value less than its breakdown voltage when $p_{\mathrm{x}}$ is negative. A large coefficient $K$ is introduced to link the relationship of carrier density at the reverse bias junction and the width of the depletion layer. However, the value is essentially subjective and nonphysical. Thus, charge conservation principle is applied to relate the side carrier density to the junction, which is expressed as:

$$
\begin{aligned}
\frac{\mathrm{d} Q}{\mathrm{~d} t}= & I_{\mathrm{mos}}+\left(C_{\mathrm{dsj}}+C_{\mathrm{gd}}\right) \frac{\mathrm{d} v_{\mathrm{ds}}}{\mathrm{d} t}-C_{\mathrm{gd}} \frac{\mathrm{d} v_{\mathrm{gs}}}{\mathrm{d} t} \\
& -\frac{Q}{\tau_{\mathrm{HL}}}-q A h_{\mathrm{p}} p_{1}^{2}
\end{aligned}
$$

where $Q$ is all the storage quantity of electric charge in the drift 
region, which can be easily obtained by the discrete value of $\mathrm{p}$ in (22). The total voltage drop of the IGBT is composed of three parts, that is the drain-source voltage of the MOSFET $v_{\mathrm{ds}}$, the forward bias voltage of $P^{+}-N^{-}$junction voltage $v_{\mathrm{j}}$, and the voltage drop of the $N^{-}$drift region $V_{N^{-}}$. The voltage drop in the lightly doped drift region $V_{N^{-}}$is comprised of two components, a resistive voltage drop due to the resistivity of the lightly doped drift region, and the Dember voltage drop due to the diffusion across the drift region, which is obtained by [24]:

$V_{N-}=\frac{I_{\mathrm{T}}}{q\left(\mu_{\mathrm{n}}+\mu_{\mathrm{p}}\right)} \frac{x_{\mathrm{r}}-x_{\mathrm{1}}}{M-1}$.

$\sum_{k=1}^{M-1}\left(\frac{1}{p_{k}-p_{k-1}} \log \frac{p_{k}}{p_{k-1}}\right)-V_{\mathrm{T}}\left(\frac{\mu_{\mathrm{n}}-\mu_{\mathrm{p}}}{\mu_{\mathrm{n}}+\mu_{\mathrm{p}}}\right) \log \left(\frac{p_{\mathrm{M}+1}}{p_{1}}\right)$

The diode is based on the same principle of IGBT just without the MOSFET part. Therefore, the IGBT and diode module forms an implicit non-linear differential-algebraic equation with carrier density, drain-source voltage $v_{d s}$, gatesource voltage $v_{g s}$, and the device current $I_{\mathrm{T}}$ is the interface with the out circuit, whose equation depends on the circuit topology. The parameters of the physics-based model are extracted via the method elaborated in [20] and given in Table II of Appendix. The implicit non-linear differential-algebraic equations are time discretized and solved numerically by the method proposed in [25]. The main advantage of the IGBT model is the elimination of the artificially introduced feedback coefficient, which is actually a non-physics factor. Therefore, the improved model becomes a pure physics-based model.

\section{B. Stray Parameter Extraction of High-Voltage IGBT Package and Sub-Module}

The introduction of advanced power devices, such as IGBTs or MOSFET, has led to the development of some packaging concept that can accommodate multiple chips for each electronic function at the same time. This structure has become the standard module design in the field of power electronics. The example in Fig. 2 illustrates the general feature of the concept: the module is composed of 6 units which are connected in parallel with the copper layer and bond wire. Each unit consists of 4 IGBT dies and 2 FWD chips with their terminal assembled reasonably. The power load current terminal is welded to the corresponding trace of the substrate. Although the welded IGBT module is the most common package for IGBTs, for applications requiring a series connection, press pack modules are preferred because of the ease with which they can be connected electrically and mechanically in series [14]. Therefore, the press pack modules are usually applied in the hybrid DC breakers so far.

The rated voltage of the IGBT module selected in most of the MMC-HVDC projects are as high as $3.3 \mathrm{kV}$, and all of them are almost in the similar package structure, whose 3D model is built in ANSYS ${ }^{\circledR} \mathrm{Q} 3 \mathrm{~d}$ and shown in Fig. 2. The stray parameters are extracted, which include parasitic resistance and inductance of the bond wire and copper track connected at the

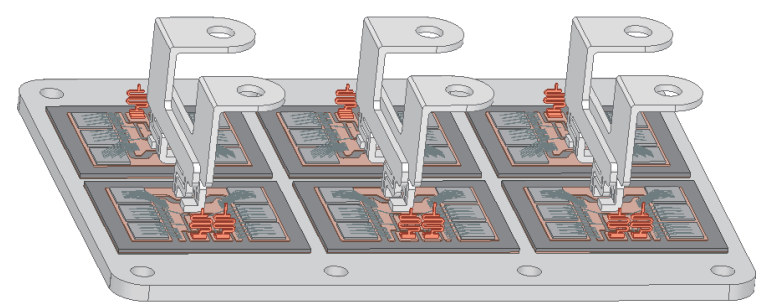

Fig. 2. IGBT package structure.

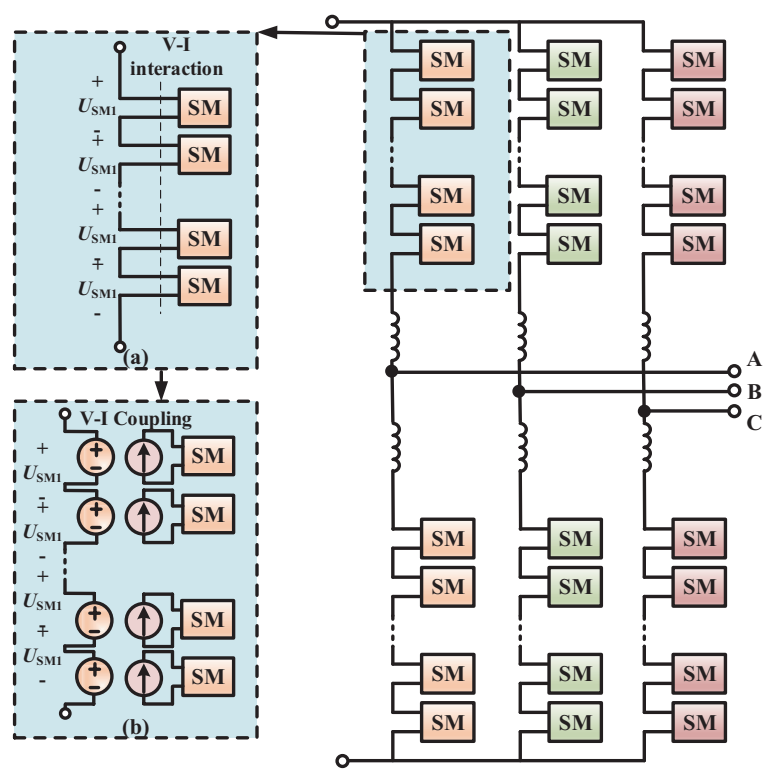

Fig. 3. Circuit partition of MMC bridge arm.

terminal of the IGBT module and the stray capacitance across the bus bars.

\section{Massive Parallel Simulation for MMC on GPU}

In this paper, the frequency scope concerned is $150 \mathrm{kHz}-30$ $\mathrm{MHz}$, thus the time-step is set to be $10 \mathrm{~ns}$ based on the theory of discrete Fourier transform. The amount of the variables to be solved in a half-bridge SM is $N_{1}+N_{2}+6$, if the space section set in the FD modeling of drift region in IGBT and diode are $N_{1}$ and $N_{2}$ respectively. For a converter with tens or even hundreds of levels, the entire system will be extremely large so that non-convergence often happens implemented on traditional simulation platforms such as Simulink with nodal voltage method or PSCAD/EMTDC with the state-space method. Even if convergence is achieved, the simulation time will often be too long to accept.

\section{A. Decoupling of $M M C$}

To optimize the solution process and achieve the urgently needed speed-up for the simulation, a large-scale parallelism method is implemented on GPU. The basic configuration of a three-phase $(\mathrm{N}+1)$-level MMC is given in Fig. 3, where the $\mathrm{N}$ cascaded SMs in each arm would pose a severe computational challenge for efficient simulation on processors if implemented 


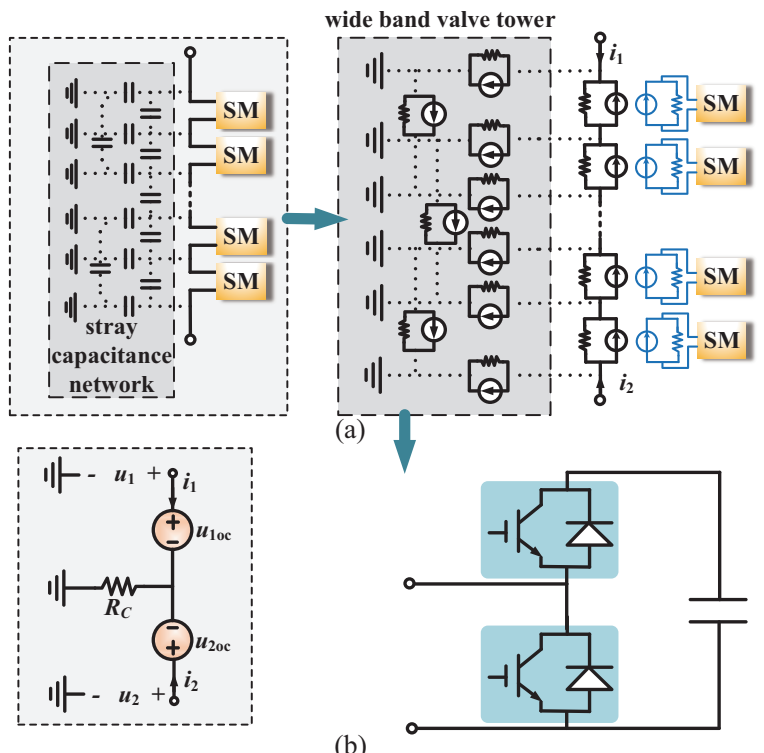

(b)

Fig. 4. Wideband model of MMC bridge arm. (a) Stray capacitance inside the converter. (b) Two-port equivalent circuit.

without simplification, particularly when the IGBT/diode nonlinear models are adopted. Compared with the switching voltage, the frequency of arm current is relatively low, which means that the current can be regarded as constant between two adjacent time steps, especially when the time-step is as small as $10 \mathrm{~ns}$. Therefore, the V-I coupling method which separates all sub-modules from their corresponding branches to form the minimum separable sub-circuit, so as to alleviate the calculation burden and improve the numerical stability, as shown in Fig. 3. In the main circuit, the MMC bridge arm collects all the SM output voltages, while the SM receives the arm current as its input. Thus, the two types of circuits conduct calculation without mutual interference until a new round of V-I interaction is completed [15].

\section{B. Large-Scale Parallel Implementation on GPU}

The distribution of the stray parameter inside an MMC is quite complicated, which not only includes the parasitics inductance and resistance of the bond wires and the connectors of the semiconductor module and busbar, but also the complex stray capacitance network shown in Fig. 4(a).

With the capacitance represented by their discretized circuit, the wideband model of the converter arm constitute a twoport network with an internal independent current source, which can be further simplified into the form shown in Fig. 4(b). The process for the transformation can achieve a highly parallel implementation. All the aforementioned algorithm is programmed on the CUDA C platform with the corresponding function module designed as a specific kernel [23]. All the kernels are executed one after another, then update and transfer the variable through the global memory. Each kernel evokes a series of threads, whose amount depends on the number of the module in the system accordingly.

The time-domain simulation starts with the system-level cal-
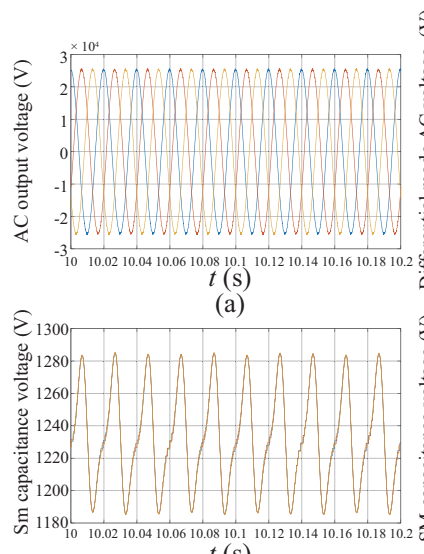

(c)

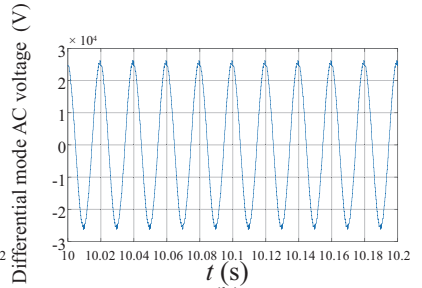

(b)

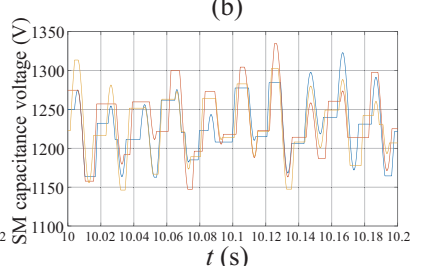

(d)
Fig. 5. System-level waveform for MMC-HVDC system. (a) AC output voltage. (b) Differential mode AC voltage. (c) SM capacitance voltage for traditional voltage sort strategy. (d) Maintaining factor-based sort strategy.

culation, in which the semiconductor devices are modeled as ideal switched, to stride over the start-up and charging process. Then the physics-based IGBT and diode models substitute for the simplified model to capture the high-frequency characteristics. The system-level waveforms are shown in Fig. 5. A novel capacitor voltage balancing method is used to reduce the switching frequency of sub-modules [26]. If the bridge arm current makes the sub-module charged, a maintaining factor slightly greater than 1 should be applied to multiply the voltage for the SM capacitance which is in the off-state and higher than the lower limit before the sorting process, so that the possibility of these SMs to keep the current off-state during the next trigger action will be increased. The strategy for the discharge situation has a similar principle. As shown in Fig. 5 (c) and (d), the switching frequency of the SMs is obviously decreased. The flowchart for the large-scale parallel simulation and the sort processing are illustrated in Fig. 6.

It has been demonstrated [15], [16] that the simulation conducted by SaberRD or PSCAD/EMTDC is no longer convergent for a device-level model once the voltage level reaches more than 11 . Thus, numerical stability and speed of convergence comparison are made between GPU and CPU based on the model proposed in this paper, which is shown in Table I. The time for CPUs and the NVIDIA Tesla V100 GPU to simulate the 2-terminal MMC-HVDC system for 2 cycles duration (0.04 s) with a time-step of $10 \mathrm{~ns}$. Obviously, compared with CPU, GPU based simulation brings significant acceleration, and with the increase of the number of modules, the growth rate of parallel simulation time is basically linear, which will greatly improve the efficiency of converter analysis and design.

\section{Valve Tower Radiated Principle and Antenna Model}

\section{A. Radiated Sources and Emission}

The semiconductor devices turn on and off rapidly during the operation of the converter, the voltage and current waveform 


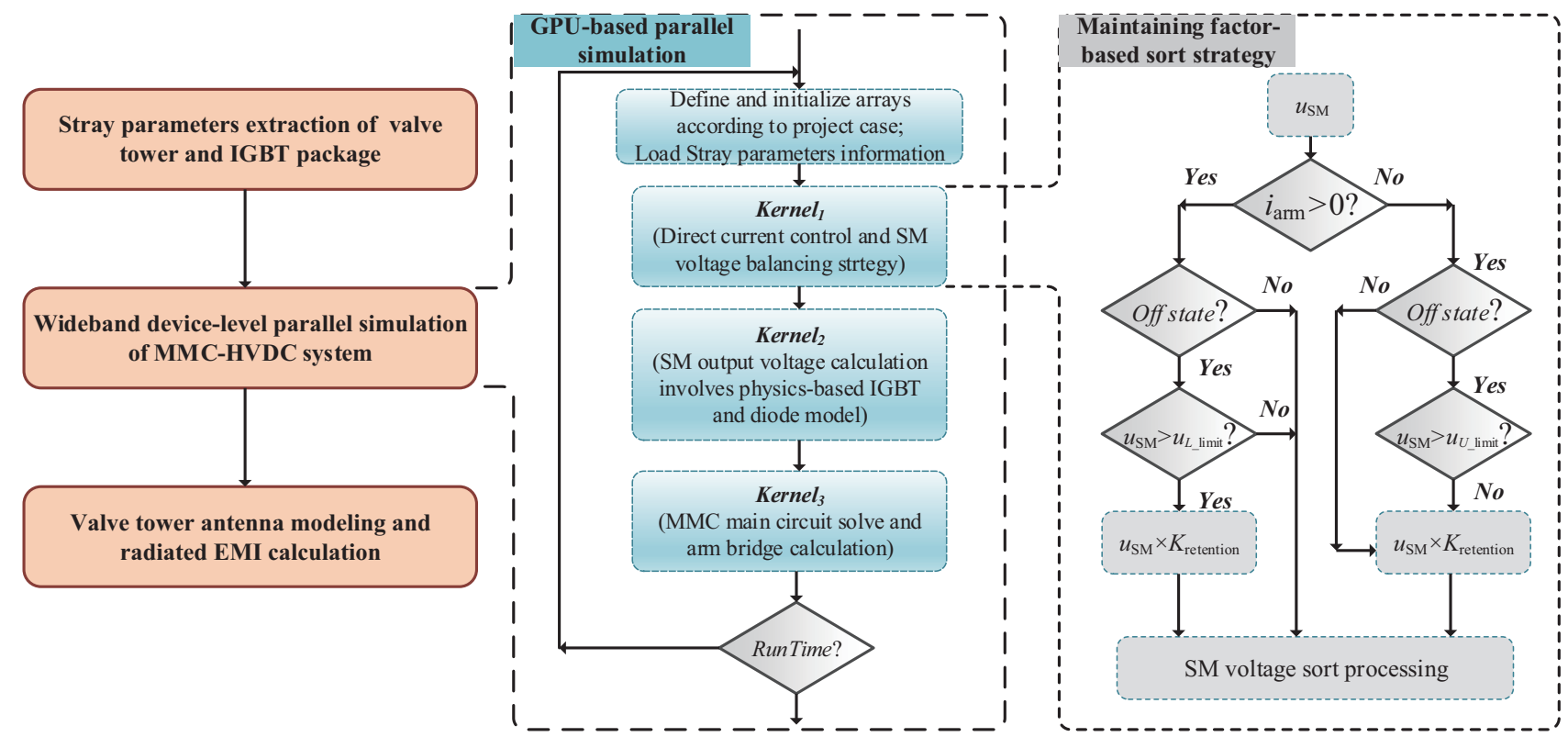

Fig. 6. Flowchart of the radiated EMI calculation.

TABLE I

ExeCUtion Time of 2-Terminal MMC-HVDC GRID By CPUs AND GPU for 2 CYCLES $(0.04 \mathrm{~s})$

\begin{tabular}{lccc}
\hline \hline \multirow{2}{*}{ MMC Level } & \multicolumn{2}{c}{ Execution Time (s) } & \multirow{2}{*}{ Speedup } \\
\cline { 2 - 3 } & $20 \mathrm{CPU}$ cores & V100 GPU & \\
\hline $21-\mathrm{L}$ & $5.31 \times 10^{4}$ & $3.70 \times 10^{3}$ & 14.35 \\
$49-\mathrm{L}$ & $1.46 \times 10^{5}$ & $3.81 \times 10^{3}$ & 38.32 \\
$101-\mathrm{L}$ & $3.17 \times 10^{5}$ & $5.70 \times 10^{3}$ & 55.61 \\
$201-\mathrm{L}$ & $6.55 \times 10^{5}$ & $8.29 \times 10^{3}$ & 79.01 \\
\hline \hline
\end{tabular}

contains a considerable amount of high-frequency component. Meanwhile, the current and voltage resonance be-tween the junction capacitance of the semiconductor devices, the stray capacitance in the module package, SM structure, and the parasitic inductance due to the bond wire and bus bar connection.

Different from conventional dipole antenna which has an identical length for two poles and is driven by a voltage source, the input and output of power connect in the power converter may have different lengths and is driven by a voltage source in series with an equivalent source impedance [27].

Each sub-module charges and discharges the capacitor continuously with the operation of the device. The small loop formed by each sub-module becomes a dipole antenna, which radiates electromagnetic waves continuously. At the same time, the copper bar connecting the sub-module itself constitutes a spiral loop, in which the high-frequency part of the current in circulation also radiates electromagnetic waves. Due to the application of the busbar which links the IGBT modules to form the submodule units, space current loop inside each $\mathrm{SM}$ is quite small so that its effect can be ignored compared with the radiation ability of the connecting copper bar in the bridge arm. Therefore, the sub-module voltage and bridge arm current are the main sources of electromagnetic interference

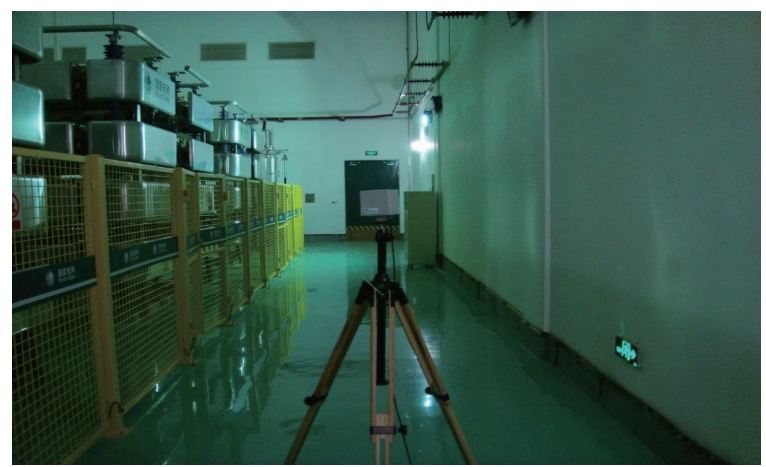

Fig. 7. Measurement of radiated EMI inside the valve hall.

radiated by converter valves. Accurate simulation calculation of sub-module charging and discharging process and bridge arm current fluctuation is very important for the evaluation of electromagnetic disturbance intensity. On the other hand, the electromagnetic wave is refracted inside the converter valve and radiates outward through the gap of the shielding plate of the converter valve tower. Therefore, the accurate modeling of the shielding system is also an important part. The cables behave like an undesired antenna at high frequencies. The high-frequency parasitics inside the converter will greatly affect the high-frequency characteristics of source impedance so that they cannot be ignored.

\section{B. Radiated EMI Measurement in Valve Hall and Model Verification}

The near-field results are calculated and compared with the actual measurement within the valve hall of an MMC-HVDC station as shown in Fig. 7, in which the ROHDE\&SCHWARZELS-3 EMI receiver $(9 \mathrm{kHz}-3 \mathrm{GHz})$ and the ZN30900 loop antenna $(10 \mathrm{kHz}-30 \mathrm{MHz})$. The converter is with $56 \mathrm{SMs}$ in each arm, 8 of which are redundant. The rate active power of the $\mathrm{MMC}$ is $18 \mathrm{MVA}$, the $\mathrm{AC}$ and $\mathrm{DC}$ side voltages are $31 \mathrm{kV}$ 


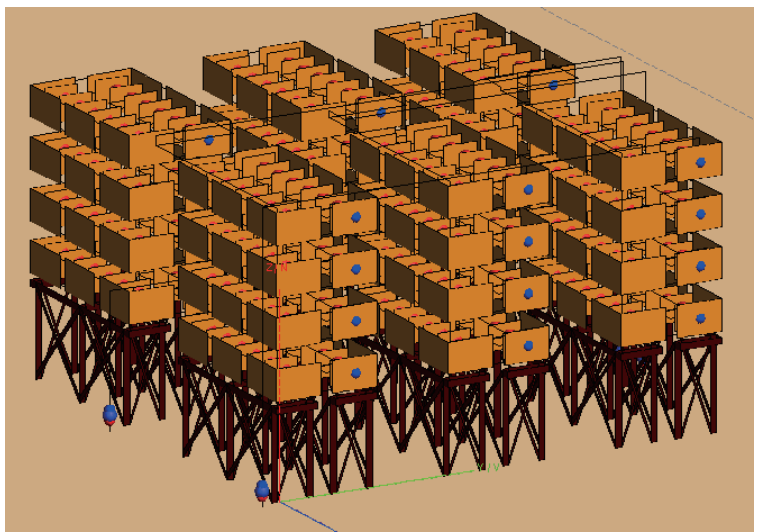

Fig. 8. 3D model established in Alteir ${ }^{\circledR}$ FEKO.

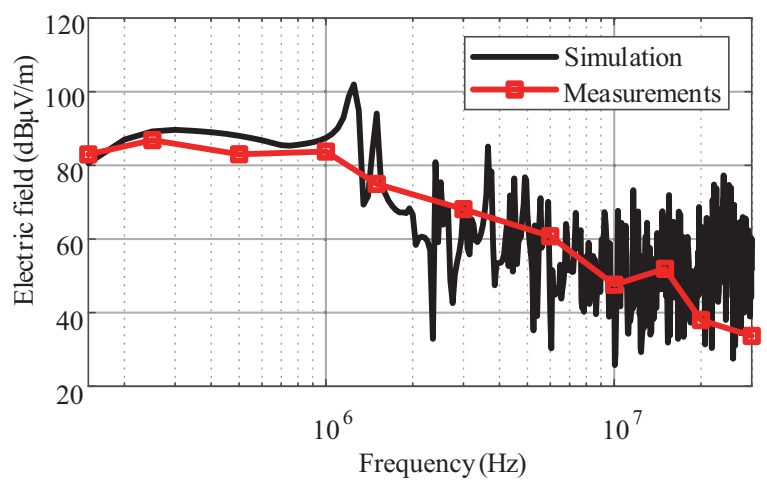

Fig. 9. Comparison of the experimental and calculated results.

and $60 \mathrm{kV}$ respectively. The bridge arm inductance and SM capacitances are $53 \mathrm{mH}$ and $6 \mathrm{mF}$, and the parameters for the control system are given in Table III in Appendix. The converter valve tower is regarded as a complex antenna according to its structure and is modeled in Alteir ${ }^{\circledR}$ FEKO as shown in Fig. 8.

Fig. 9 shows the comparison of measured and simulated results. It can be seen that the EMI level decreases with the increase of frequency, with a peak value near $1 \mathrm{MHz}$ and 2 $\mathrm{MHz}$. Both the calculated spatial electric field intensity and the measured frequency point are in the same range and have the same change trend, which proves the correctness and effectiveness of the method proposed in this paper. Thus the model is competent for MMC-HVDC engineering design guidance work.

The electromagnetic environment of the inspection corridor directly affects the physiological safety of the operation and maintenance engineers. Thus, the prediction and analysis of the electric field distribution of the inspection corridor under the normal condition of the valve tower are essential. The electric field distribution of each typical frequency point located within the inspection corridor is shown in Fig. 10.

The maximum and minimum magnitude of the electric field for the $1 \mathrm{MHz}$ component is $68.5 \mathrm{dBV} / \mathrm{m}$ and $8.5 \mathrm{dBV} / \mathrm{m}$. For some other frequency points $(6 \mathrm{MHz}, 10 \mathrm{MHz}, 15 \mathrm{MHz}, 20$ $\mathrm{MHz}$, and $30 \mathrm{MHz}$ ), the electric field intensity are between

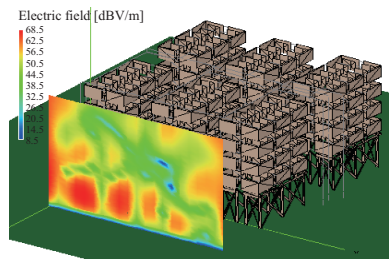

(a)

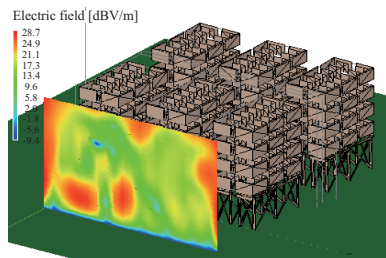

(c)

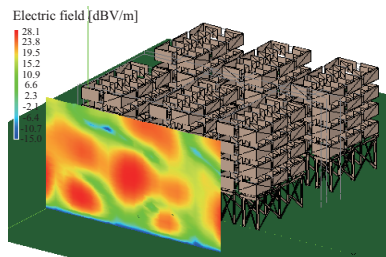

(e)

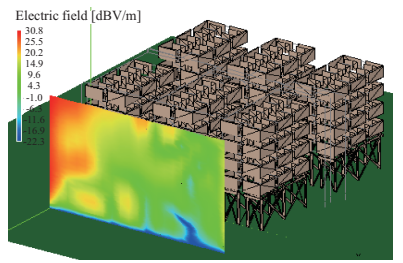

(b)

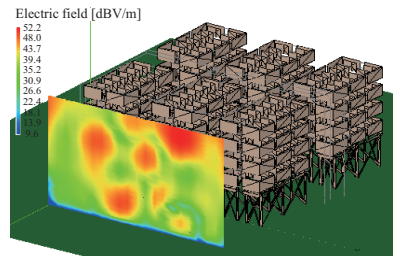

(d)

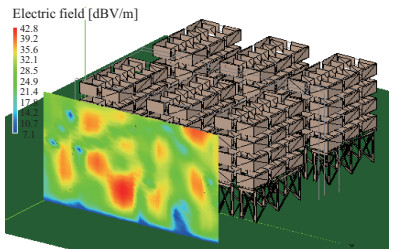

(f)
Fig. 10. The E-field distribution for components of: (a) $1 \mathrm{MHz}$, (b) $6 \mathrm{MHz}$, (c) $10 \mathrm{MHz}$, (d) $15 \mathrm{MHz}$, (e) $20 \mathrm{MHz}$, and (f) $30 \mathrm{MHz}$.

$-22.3 \mathrm{dBV} / \mathrm{m}$ and $30.8 \mathrm{dBV} / \mathrm{m},-9.4 \mathrm{dBV} / \mathrm{m}$ and $28.7 \mathrm{dBV} /$ $\mathrm{m}, 9.6 \mathrm{dBV} / \mathrm{m}$ and $52.2 \mathrm{dBV} / \mathrm{m},-15 \mathrm{dBV} / \mathrm{m}$ and $28.1 \mathrm{dBV} / \mathrm{m}$, $7.1 \mathrm{dBV} / \mathrm{m}$ and $42.8 \mathrm{dBV} / \mathrm{m}$ respectively. The maximum value of electric field intensity tends to decrease with the increase of frequency. While near the A-phase (leftmost) valve towers in Fig. 10 at $1 \mathrm{MHz}, 6 \mathrm{MHz}$ and $10 \mathrm{MHz}$, and in front of the B-phase (middle) valve towers for other frequency points, the corresponding values are relatively large.

The simulation program and the antenna model are modified to calculate the radiated EMI generated by a $21-\mathrm{L}$ MMCHVDC valve tower, and compared with the original 49-L converter under the output connectors at both AC and DC side. The results are shown in Fig. 11. It can be concluded that the EMI will be suppressed to some extent within the highfrequency scope (above $2 \mathrm{MHz}$ ) through an increase of the level, however, the attendant side effect is a remarkable growth of the EMI level in the low-frequency range especially for the DC side, which is due to the more oscillation introduce by more SMs. Thus, particular attention should be paid when the decision for the amount of the SMs are made during the converter EMI validation in the design stage.

Fig. 12 shows the result for the low switching frequency situation at the same measurement point as Fig. 7 when the maintaining factor-based SM capacitance voltage sort strategy is applied. Compared with the traditional voltage balancing method with a high switching frequency, the radiated EMI level will be reduced within the scope between $150 \mathrm{kHz}$ and 1 $\mathrm{MHz}$ when the SMs are operated with less frequent commutations. Therefore, some voltage sorting method with reduced switching frequency can be considered as an optimization method for EMI suppression. 


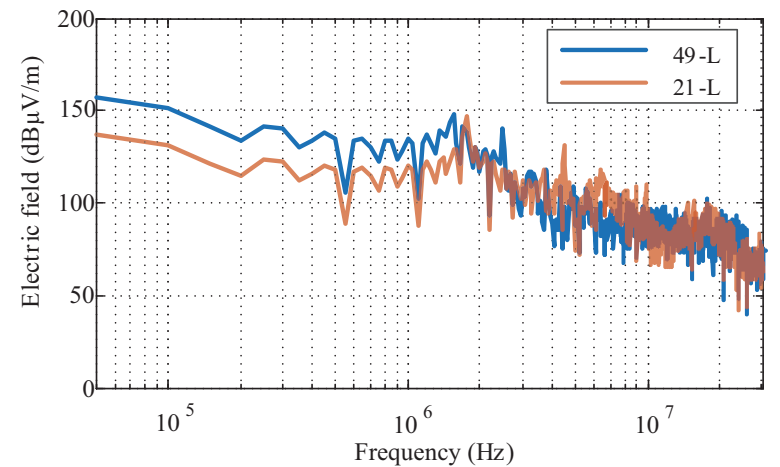

(a)

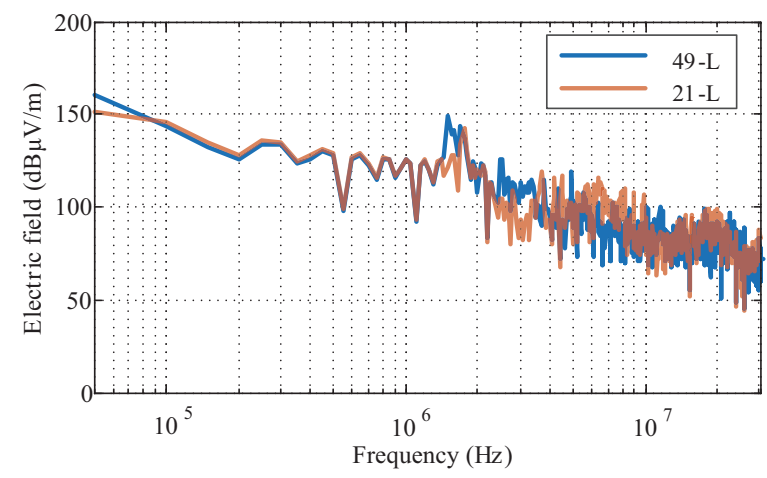

(b)

Fig. 11. Radiated EMI with different levels. (a) DC side. (b) AC side.

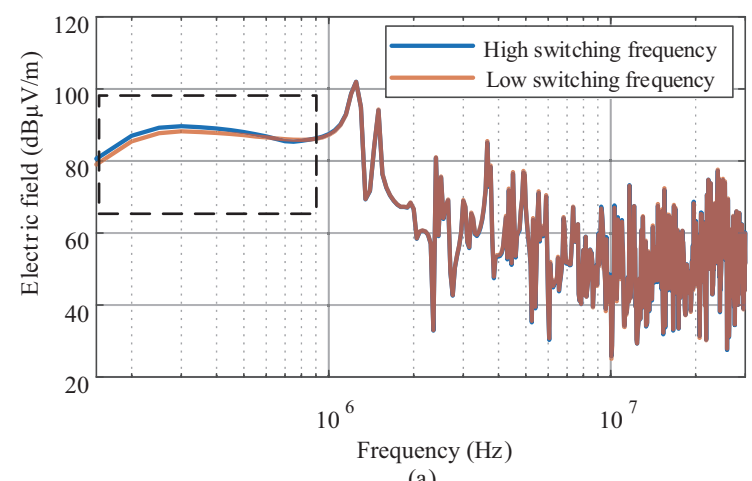

(a)

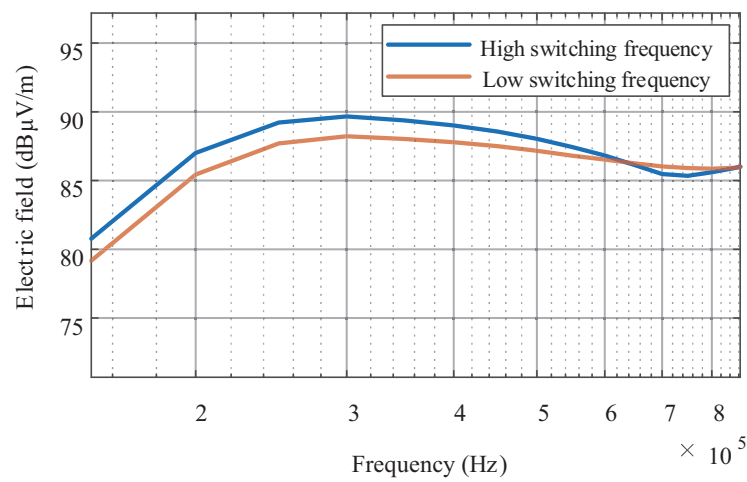

(b)

Fig. 12. Radiated EMI with different SM voltage balancing strategy. (a) Comparison of the radiated EMI. (b) Zoom-in plot of the low-frequency scope.
TABLE II

Parameters of Physics-Based IGBT and Diode Model
TABLE III

Parameters of Control System for MMC-HVDC Project

$K p_{\mathrm{dc}}=0.2, K i_{\mathrm{dc}}=1, K p_{\mathrm{Q}}=0.2, K i_{\mathrm{Q}}=1$

$K p_{\mathrm{d}}=0.6, K i_{\mathrm{d}}=6, K p_{\mathrm{q}}=0.6, K i_{\mathrm{q}}=6$

\section{Conclusion}

This paper presents a method that can achieve the prediction of electromagnetic interference in the valve hall in the design stage of the MMC-HVDC converter. The independence of the actual measurement renders the prediction of the radiated EMI divorced from the tedious and uneconomical of trial and error process. Based on the wide-band time-domain simulation of the converter, the current of the valve tower connected with the copper bar is used as the radiator, and the output voltage of the submodule is used as the multiple excitation sources of the equivalent antenna. By building a three-dimensional antenna model in FEKO, the calculation of the electromagnetic interference radiated by the converter is carried out. The timedomain simulation of the converter involves the physics-based transient characteristics of the power semiconductor devices, the control strategy of the system, and all the stray parameters. The related calculation burden is alleviated completely via parallel processing by voltage and current decoupling method, which avoids the large-scale matrix iterative solution, greatly shortens the simulation time. The analysis and verification of the final results not only prove the correctness of the method but also predict that the influence of the capacitor voltage balancing strategy on radiated EMI is limited to the lower frequency scope.

\section{APPENDIX}

The parameters of physics-based IGBT and diode model are given in Table II.

The parameters of control system for MMC-HVDC project are given in Table III.

\section{REFERENCES}

[1] E. Sánchez-Sánchez, E. Prieto-Araujo, A. Junyent-Ferré, V. JaliliMarandi, and O. Gomis-Bellmunt, "Analysis of MMC energy-based control structures for VSC-HVDC links," in IEEE Journal of Emerging and Selected Topics in Power Electronics, vol. 6, no. 3, pp. 1065-1076, 
Sep. 2018

[2] C. Zou, H. Rao, S. Xu, Y. Li, W. Li, J. Chen, X. Zhao, Y. Yang, and B. Lei, "Analysis of resonance between a VSC-HVDC converter and the AC grid," in IEEE Transactions on Power Electronics, vol. 33, no. 12, pp. 10157-10168, Dec. 2018.

[3] G. Li, D. Zhang, Y. Xin, S. Jiang, W. Wang, and J. Du, "Design of MMC hardware-in-the-loop platform and controller test scheme," in CPSS Transactions on Power Electronics and Applications, vol. 4, no. 2, pp. 143-151, Jun. 2019.

[4] A. Christe, M. Petkovic, I. Polanco, M. Utvic, and D. Dujic, "Auxiliary submodule power supply for a medium voltage modular multilevel converter," in CPSS Transactions on Power Electronics and Applications, vol. 4, no. 3, pp. 204-218, Sep. 2019.

[5] F. Costa and D. Magnon, "Graphical analysis of the spectra of EMI sources in power electronics," in IEEE Transactions on Power Electronics, vol. 20, no. 6, pp. 1491-1498, Nov. 2005.

[6] V. S. Nguyen, P. Lefranc, and J. C. Crebier, "Gate driver supply architectures for common mode conducted EMI reduction in series connection of multiple power devices," in IEEE Transactions on Power Electronics, vol. 33, no. 12, pp. 12265-10276, Sep. 2018.

[7] J. Zhang, T. Lu, W. Zhang, J. Xu, and W. Li, "Measurement and analysis of radiated disturbance characteristics of $\pm 320 \mathrm{kV}$ modular multilevel converter system," in IEEE Access, vol. 7, pp. 10028-10036, Jan. 2019.

[8] J. Zhang, T. Lu, W. Zhang, H. Shen, and Z. Yang, "Frequency-time domain characteristics of radiated electric fields in a multi-terminal MMC-HVDC station," in IEEE Access, vol. 7, pp. 99937-99944, Aug. 2019.

[9] M. M. Hernando, A. Fernández, M. Arias, M. Rodríguez, Y. Á lvarez, and F. Las-Heras, "EMI radiated noise measurement system using the source reconstruction technique," in IEEE Transactions on Industrial Electronics, vol. 55, no. 9, pp. 3258-3265, Sep. 2008.

[10] A. Nejadpak, A. Sarikhani, and O. A. Mohammed, "Analysis of radiated EMI and noise propagation in three-phase inverter system operating under different switching patterns," in IEEE Transactions on Magnetics, vol. 49, no. 5, pp. 2213-2216, May 2013.

[11] Z. Yu, J. He, R. Zeng, H. Rao, X. Li, Q. Wang, B. Zhang, and S.Chen, "Simulation analysis on conducted EMD caused by valves in $\pm 800 \mathrm{kV}$ UHVDC converter station," in IEEE Transactions on Electromagnetic Compatibility, vol. 51, no. 2, pp. 236-244, May 2009.

[12] Y. Huangfu, S. Wang, L. D. Rienzo, and J. Zhu, "Radiated EMI modeling and performance analysis of a PWM PMSM drive system based on field-circuit coupled FEM," in IEEE Transactions on Magnetics, vol. 53, no. 11, pp. 1-4, Nov. 2017.

[13] I. F. Kovačević, T. Friedli, A. M. Müsing, and J. W. Kolar, "3-D electromagnetic modeling of parasitics and mutual coupling in EMI filters," in IEEE Transactions on Power Electronics, vol. 29, no. 1, pp. 135-149, Jan. 2014.

[14] Power semiconductors product brochure 2017, ABB Corp., Jun. 2017.

[15] N. Lin and V. Dinavahi, "Exact nonlinear micro-modeling for finegrained parallel EMT simulation of MTDC grid interaction with wind farm," in IEEE Transactions on Industrial Electronics, vol. 66, no. 8, pp. 6247-6436, Aug. 2019

[16] S. Yan, Z. Zhou, and V. Dinavahi, "Large-scale nonlinear device level power electronic circuit simulation on massively parallel graphics processing architectures," in IEEE Transactionson Power Electronics, vol. 33, no. 6, pp. 4660-4678, Jun. 2018.

[17] A. R. Hefner and D. M. Diebolt, "An experimentally verifed IGBT model implemented in the Saber circuit simulator," in IEEE Transactions on Power Electronics, vol. 9, no. 5, pp. 532-542, Sep. 1994.

[18] A. R. Hefner, "Analytical modeling of device-circuit interactions for the power insulated gate bipolar transistor(IGBT)," in IEEE Transactions on Industry Applications, vol. 26, no. 6, pp. 995-1005, Nov. 1990.

[19] A. R. Hefner, "An improved understanding for the transient operation of the power insulated gate bipolar transistor(IGBT)," in IEEE Transactions on Power Electronics, vol. 5, no. 4, pp. 459-468, Oct. 1990.

[20] A. T. Bryant, X. Kang, E. Santi, P. R. Palmer, and J. L. Hudgins, "Twostep parameter extraction procedure with formal optimization for physics-based circuit simulator IGBT and p-i-n diode models," in IEEE
Transactions on Power Electronics, vol. 21, no. 2, pp. 295-309, Mar. 2006.

[21] R. Chibante, A. Araujo, and A. Carvalho, "Finite-element modeling and optimization-based parameter extraction algorithm for NPTIGBTS," in IEEE Transactions on Power Electronics, vol. 24, no. 5, pp. 1417-1427, May 2009.

[22] G. Buiatti, F. Cappelluti, and G. Ghione, "Physics-based PiN diode SPICE model for power-circuit simulation," in IEEE Transactions on Industry Applications, vol. 43, no. 4, pp. 911-919, Jul. 2007.

[23] CUDA C programming guide, NVIDIA Corp., Jun. 2017.

[24] T. K. Gachovska, J. L. Hudgins, E. Santi, A. Bryant, and P. R. Palmer, Modeling Bipolar Power Semiconductor Devices, Jun. 2013.

[25] L. F. Shampine, "solving $0=\mathrm{F}(\mathrm{t}, \mathrm{y}(\mathrm{t}), \mathrm{y}$ '(t))in Matlab," in Journal of Numerical Mathematics, vol. 10, no. 4, pp. 291-310, Jul. 2002.

[26] M. Guan and Z. Xu, "Optimized capacitor voltage balancing control for modular multilevel converter based VSC-HVDC system," in Proceedings of the CSEE (in Chinese), vol. 31, no. 12, pp. 9-14, Apr. 2011.

[27] Y. Zhang, S. Wang, and Y. Chu, "Investigation of radiated electromagnetic interference for an isolated high-frequency DC-DC power converter with power cables," in IEEE Transactionson Power Electronics, vol. 34, no. 10, pp. 295-309, Oct. 2019.

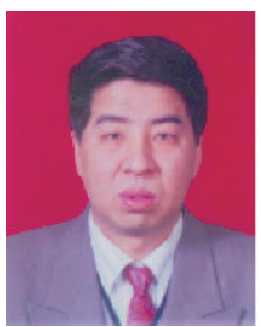

Guishu Liang received the B.S., M.S., and Ph.D. degrees in electric engineering from North China Electric Power University, China, in 1982, 1987, and 2008, respectively, where he is currently a Professor and a Doctoral Tutor and also serves as the Director of the Electrical Technician Department. His research interests include electrical theory and new technology.

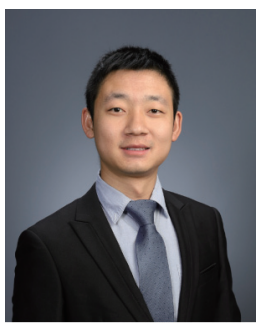

Ruimin Zhu received the B.Eng. degree in electrical engineering from North China Electric Power University, Baoding, Hebei, China, in 2012. He is currently pursuing his Ph.D. degree at North China Electric Power University, Beijing, China. In 2018 and 2019, he is a Joint Ph.D. Student in RTXLAB, University of Alberta, Edmonton, AB, Canada. His research interests include EMI analysis of power systems, power electronics, device-level modeling and parallel simulation. 